\title{
Precise measurements of the dipole moment and polarizability of the neutral exciton and positive trion in a single quantum dot
}

\author{
J. D. Mar, ${ }^{1,2, *}$ J. J. Baumberg, ${ }^{2}$ X. L. Xu,$^{1, \dagger}$ A. C. Irvine, ${ }^{3}$ and D. A. Williams ${ }^{1}$ \\ ${ }^{1}$ Hitachi Cambridge Laboratory, Cavendish Laboratory, Cambridge CB3 OHE, United Kingdom \\ ${ }^{2}$ NanoPhotonics Centre, Cavendish Laboratory, University of Cambridge, Cambridge CB3 OHE, United Kingdom \\ ${ }^{3}$ Microelectronics Research Centre, Cavendish Laboratory, University of Cambridge, Cambridge CB3 OHE, United Kingdom
}

(Received 4 April 2017; revised manuscript received 9 May 2017; published 23 May 2017)

\begin{abstract}
We perform precise measurements of the permanent dipole moment and polarizability of both the neutral exciton $\left(X^{0}\right)$ and positive trion $\left(X^{+}\right)$in a single InAs/GaAs self-assembled quantum dot (QD). This is achieved through one- and two-color high-resolution photocurrent (PC) spectroscopy of $X^{0}$ and $X^{+}$, respectively, using ultra-narrow-bandwidth continuous-wave lasers. This technique allows for sub- $\mu \mathrm{eV}$ resolution, which is limited only by the spectral linewidth of the lasers and is more than four orders of magnitude higher than that of previous techniques. We are therefore permitted to obtain precise values for the permanent dipole moment and polarizability of both $X^{0}$ and $X^{+}$, by fitting an appropriate theoretical model to the measured transition energies as a function of electric field. As a sequence of protocols for the optical initialization, manipulation, and readout of a QD hole spin qubit embedded in a photodiode device relies on the coherent control of both $X^{0}$ and $X^{+}$as intermediary states, such precise measurements of their dipole moment and polarizability using high-resolution PC spectroscopy are crucial for implementing these quantum computing protocols with high fidelity.
\end{abstract}

DOI: 10.1103/PhysRevB.95.201304

The spin of an electron or hole confined in a semiconductor quantum dot (QD) is a promising candidate for a solid-state qubit due to its long relaxation and decoherence times [1-3] and scalability into large arrays. Virtually all protocols for the optical initialization [4-7], manipulation [8-10], and readout $[6,11,12]$ of a QD spin qubit rely on intermediary exciton states in order to perform the desired quantum computing operation. A particular sequence of such protocols that has attracted much interest recently $[6,7,13,14]$ relies on the coherent control of both the neutral exciton $\left(X^{0}\right)$ and positive trion $\left(X^{+}\right)$states as intermediary states in order to perform initialization, manipulation, and readout of a QD hole spin qubit embedded in a photodiode device. Therefore, precise measurements of the permanent dipole moment (i.e., Rabi frequency) and polarizability of both $X^{0}$ and $X^{+}$using highresolution photocurrent (PC) techniques are crucial for executing such quantum computing protocols with high fidelity. To date, such measurements have been lacking in the scientific literature and the techniques used by other groups $[6,15]$ prohibit such precise measurements due to a considerably low resolution, which is determined by various factors such as the spectral linewidth $(\sim 0.2 \mathrm{meV})$ of transform-limited laser pulses.

Here, we perform precise measurements of the permanent dipole moment and polarizability of both $X^{0}$ and $X^{+}$in a single InAs/GaAs self-assembled QD. This is achieved through one- and two-color high-resolution PC spectroscopy of $X^{0}$ and $X^{+}$, respectively, using ultra-narrow-bandwidth $(\sim 1 \mathrm{MHz})$ continuous-wave $(\mathrm{cw})$ lasers. This technique allows for sub- $\mu \mathrm{eV}$ resolution, which is limited only by the spectral

\footnotetext{
*Author to whom correspondence should be addressed: jm585@cam.ac.uk

${ }^{\dagger}$ Present address: Beijing National Laboratory for Condensed Matter Physics, Institute of Physics, Chinese Academy of Sciences, Beijing 100190, People's Republic of China.
}

linewidth of the lasers and is more than four orders of magnitude higher than that of previous techniques $[6,15]$. We are therefore permitted to obtain precise values for the permanent dipole moment and polarizability of both $X^{0}$ and $X^{+}$in the vertical (growth) direction, by fitting a theoretical model to the measured transition energies as a function of vertical electric field.

The device used in this Rapid Communication was designed for single-QD PC measurements and fabricated as an $n-i$ Schottky photodiode structure based on a two-dimensional electron gas (2DEG). A single layer of InAs/GaAs selfassembled QDs, which was grown to yield a low surface density of QDs $\left(\sim 10^{9} \mathrm{~cm}^{-2}\right)$, was embedded in a $250-\mathrm{nm}-$ thick layer of $i$-GaAs and positioned $50 \mathrm{~nm}$ above the $\mathrm{Si}$ $\delta$-doped GaAs layer $\left(N_{d}=5 \times 10^{12} \mathrm{~cm}^{-2}\right)$ from which the $2 \mathrm{DEG}$ is derived and confined in the resultant $\mathrm{V}$-shaped potential well $[16,17]$. In order to perform single-QD PC measurements, submicrometer-sized apertures were etched into an $\mathrm{Al}$ shadow mask via electron-beam lithography to isolate single QDs. In addition, $\mathrm{Cr} / \mathrm{Au}$ bond pads were formed on both the Schottky contact and the ohmic contact (which was made to the 2DEG) to allow for electrical connection of the device to an external voltage source and current meter. Further details of the device structure can be found in Ref. [7]. Prior to single-QD PC measurements, we first performed electric-field-dependent microphotoluminescence ( $\mu$-PL) spectroscopy in order to promptly locate isolated single QDs, measure straightforwardly the transition energies of their various excitonic states (mostly importantly, the transition energies of $X^{0}$ and $X^{+}$), and determine the electric field range within which a single-QD PC signal may be expected as a result of $e-h$ pair ionization and tunneling out from the QD following resonant laser excitation of the $X^{0}$ and $X^{+}$transitions $[18,19]$. All measurements in this work were performed at a sample temperature of $4.2 \mathrm{~K}$.

The $X^{0}$ absorption spectrum is measured using onecolor high-resolution PC spectroscopy, where resonant optical 


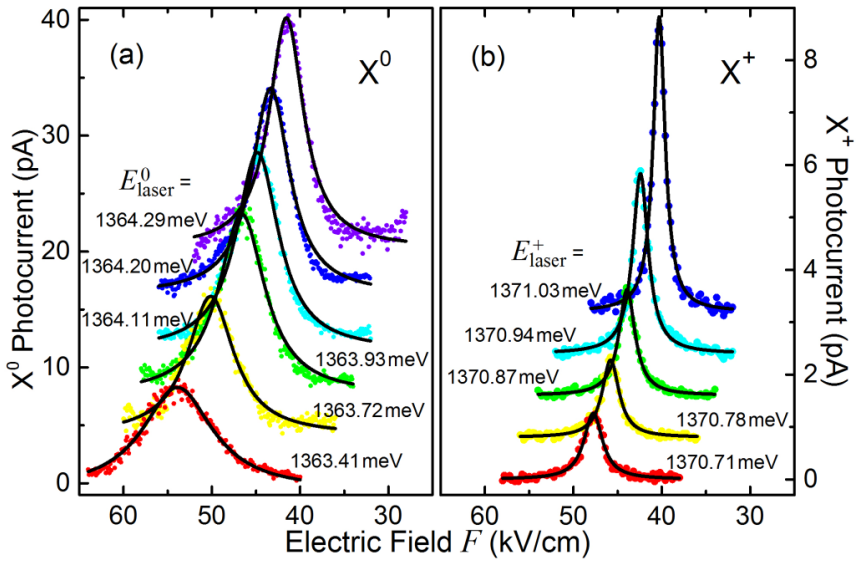

FIG. 1. Single-QD PC spectra of (a) $X^{0}$ and (b) $X^{+}$for a series of values of (a) $E_{\text {laser }}^{0}$ and (b) $E_{\text {laser }}^{+}$with Lorentzian fit curves (solid lines). For clarity, spectra are shifted vertically with respect to each other.

excitation using an ultra-narrow-bandwidth $(\sim 1 \mathrm{MHz}) \mathrm{cw}$ laser is followed by electron and hole tunneling out of the QD and towards the 2DEG and Schottky contact, respectively, under high vertical electric fields, resulting in a measurable PC signal across the photodiode. A spectrum of $X^{0}$ can thus be obtained by sweeping its transition energy through a fixed laser energy $E_{\text {laser }}^{0}$ via the quantum-confined Stark effect (QCSE), which is in turn controlled by the vertical electric field $F$ [19]. On the other hand, the $X^{+}$absorption spectrum is measured using two-color high-resolution PC spectroscopy, where an ultra-narrow-bandwidth $\mathrm{cw}$ laser is tuned to each of the $X^{0}$ and $X^{+}$transition energies. With the two lasers cross-circularly polarized, the $X^{0}$ and $X^{+}$transition energies are then swept through their respective laser energies $\left(E_{\text {laser }}^{0}\right.$ and $E_{\text {laser }}^{+}$) roughly simultaneously via the QCSE. $X^{0}$ excitation followed by fast ionization via electron tunneling allows for the possible excitation of $X^{+}$, resulting in a total PC signal consisting of an $X^{0}$ and $X^{+}$PC component after all carriers have tunneled out of the QD. As the $X^{+}$absorption peak is swept through $E_{\text {laser }}^{+}$, the excitation of $X^{+}$is dependent not only on the absorption strength of $X^{+}$at $E_{\text {laser }}^{+}$, but also on the absorption strength of $X^{0}$ at $E_{\text {laser }}^{0}$, for a given $F$. Therefore, a PC measurement of the $X^{+}$spectrum as a function of $F$ is obtained by subtracting the $X^{0}$ PC component from the total PC signal as a function of $F$, then dividing the result by the $X^{0}$ excitation probability [20].

By fitting a Lorentzian curve to the PC peak of $X^{0}\left(X^{+}\right)$, the corresponding values of $F$ and $X^{0}\left(X^{+}\right)$transition energy, which is precisely known from the value of $E_{\text {laser }}^{0}\left(E_{\text {laser }}^{+}\right)$, can be obtained. Then, by repeating the above measurement for a series of distinct values of $E_{\text {laser }}^{0}$ throughout the $F$ range within which a measurable $X^{0}$ PC peak can be obtained [Fig. 1(a)] and for a series of distinct values of $E_{\text {laser }}^{+}$throughout the $F$ range within which a measurable $X^{+}$PC peak can be obtained [Fig. 1(b)], a set of corresponding values of $F$ and transition energy is produced for each of $X^{0}$ and $X^{+}$, as plotted in Fig. 2. A set of corresponding values of $F$ and $X^{0}\left(X^{+}\right)$transition energy using $\mu$-PL spectroscopy is also plotted in Fig. 2 and was acquired by extracting values of the peak energy from

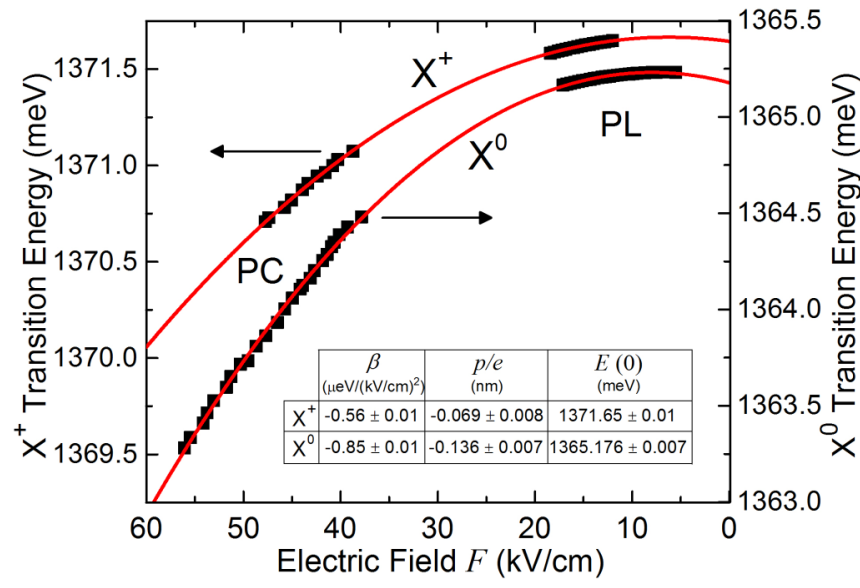

FIG. 2. Plot of the $X^{0}$ and $X^{+}$transition energies as a function of $F$ obtained through both $\mathrm{PC}$ and $\mu$-PL spectroscopy. Equation (1) is fit to the experimental data (solid lines), yielding precise values for $p, \beta$, and $E(0)$, which are given in the table in the inset.

Lorentzian curve fits to the $\mu$-PL spectra of $X^{0}\left(X^{+}\right)$for known values of $F[19,20]$. The exciton transition energy can be expressed as a function of $F$ as [18,21]

$$
E(F)=E(0)-p F+\beta F^{2},
$$

where $E(0)$ is the exciton transition energy at $F=0, p$ is the permanent dipole moment of the exciton in the vertical (growth) direction, and $\beta$ is the polarizability of the exciton in the vertical direction. As shown in Fig. 2, fitting this model to the experimental data yields extremely precise values for $p$ and $\beta$ of both $X^{0}$ and $X^{+}$, which are given in the table in the inset. While these values are comparable to those in previous works [21,22], they have a higher precision by at least an order of magnitude. It is worth noting that the slight decrease of $p$ for $X^{+}$relative to $X^{0}$ suggests that the centers of gravity of the electron and hole wave functions along the vertical direction move closer when a hole is added to the QD. Also, the negative sign of $p$ for both $X^{0}$ and $X^{+}$indicates that the center of gravity of the electron wave function is localized above that of the hole in the QD for both exciton states. This dipole alignment is consistent with theoretical calculations on pure InAs self-assembled QDs with a pyramidal shape [23-26] and is opposite to those in other works on QDs showing an inverted alignment $[21,22]$ that is due to In enrichment towards the QD apex [21]. We note that the above results for this particular QD are similar to those observed for several other QDs on the same sample.

We have performed precise measurements of $p$ and $\beta$ for both $X^{0}$ and $X^{+}$in a single InAs/GaAs self-assembled QD. This was achieved through one- and two-color highresolution PC spectroscopy of $X^{0}$ and $X^{+}$, respectively, using ultra-narrow-bandwidth $(\sim 1 \mathrm{MHz}) \mathrm{cw}$ lasers. This technique allowed for sub- $\mu \mathrm{eV}$ resolution, which is limited only by the spectral linewidth of the lasers and is more than four orders of magnitude higher than that of previous techniques $[6,15]$. We were therefore permitted to obtain precise values for $p$ and $\beta$ for both $X^{0}$ and $X^{+}$, by fitting an appropriate theoretical model to the measured transition energies as a function of vertical electric field. A sequence of protocols 
$[6,7,13,14]$ for the optical initialization, manipulation, and readout of a QD hole spin qubit embedded in a photodiode device relies on the coherent control of both $X^{0}$ and $X^{+}$as intermediary states. Therefore, while QD hole spins should be more favorable as qubits compared to electron spins since their hyperfine interaction with the nuclear spin ensemble leading to decoherence is greatly suppressed [27], such precise measurements of $p$ and $\beta$ for both $X^{0}$ and $X^{+}$using highresolution $\mathrm{PC}$ spectroscopy are crucial for implementing these protocols with high fidelity in a QD spin-based quantum computer.

The authors gratefully acknowledge Professor Colin R. Stanley (University of Glasgow) for providing the wafers. J.D.M. would like to thank the Higher Education Funding Council for England (HEFCE), the Cambridge Commonwealth Trust (CCT), and the Natural Sciences and Engineering Research Council (NSERC) of Canada for financial support.
[1] D. Loss and D. P. DiVincenzo, Phys. Rev. A 57, 120 (1998).

[2] A. Imamoglu, D. D. Awschalom, G. Burkard, D. P. DiVincenzo, D. Loss, M. Sherwin, and A. Small, Phys. Rev. Lett. 83, 4204 (1999).

[3] Semiconductor Spintronics and Quantum Computation, edited by D. D. Awschalom, D. Loss, and N. Samarth (Springer, Berlin, 2002).

[4] M. Atatüre, J. Dreiser, A. Badolato, A Högele, K. Karrai, and A. Imamoglu, Science 312, 551 (2006).

[5] B. D. Gerardot, D. Brunner, P. A. Dalgarno, P. Öhberg, S. Seidl, M. Kroner, K. Karrai, N. G. Stoltz, P. M. Petroff, and R. J. Warburton, Nature (London) 451, 441 (2008).

[6] A. J. Ramsay, S. J. Boyle, R. S. Kolodka, J. B. B. Oliveira, J. Skiba-Szymanska, H. Y. Liu, M. Hopkinson, A. M. Fox, and M. S. Skolnick, Phys. Rev. Lett. 100, 197401 (2008).

[7] J. D. Mar, J. J. Baumberg, X. L. Xu, A. C. Irvine, and D. A. Williams, Phys. Rev. B 90, 241303(R) (2014).

[8] J. Berezovsky, M. H. Mikkelsen, N. G. Stoltz, L. A. Coldren, and D. D. Awschalom, Science 320, 349 (2008).

[9] D. Press, T. D. Ladd, B. Zhang, and Y. Yamamoto, Nature (London) 456, 218 (2008).

[10] K. De Greve, P. L. McMahon, D. Press, T. D. Ladd, D. Bisping, C. Schneider, M. Kamp, L. Worschech, S. Höfling, A. Forchel, and Y. Yamamoto, Nat. Phys. 7, 872 (2011).

[11] J. Berezovsky, M. H. Mikkelsen, O. Gywat, N. G. Stoltz, L. A. Coldren, and D. D. Awschalom, Science 314, 1916 (2006).

[12] M. Atatüre, J. Dreiser, A. Badolato, and A. Imamoglu, Nat. Phys. 3, 101 (2007).

[13] T. M. Godden, J. H. Quilter, A. J. Ramsay, Y. Wu, P. Brereton, S. J. Boyle, I. J. Luxmoore, J. Puebla-Nunez, A. M. Fox, and M. S. Skolnick, Phys. Rev. Lett. 108, 017402 (2012).
[14] P.-L. Ardelt, T. Simmet, K. Müller, C. Dory, K. A. Fischer, A. Bechtold, A. Kleinkauf, H. Riedl, and J. J. Finley, Phys. Rev. B 92, 115306 (2015).

[15] K. Müller, A. Bechtold, C. Ruppert, C. Hautmann, J. S. Wildmann, T. Kaldewey, M. Bichler, H. J. Krenner, G. Abstreiter, M. Betz, and J. J. Finley, Phys. Rev. B 85, 241306(R) (2012).

[16] K. Ploog, J. Cryst. Growth 81, 304 (1987).

[17] L. M. R. Scolfaro, R. P. Camata, J. M. V. Martins, and J. R. Leite, Superlattices Microstruct. 12, 203 (1992).

[18] J. D. Mar, X. L. Xu, J. J. Baumberg, F. S. F. Brossard, A. C. Irvine, C. Stanley, and D. A. Williams, Phys. Rev. B 83, 075306 (2011).

[19] J. D. Mar, X. L. Xu, J. J. Baumberg, A. C. Irvine, C. Stanley, and D. A. Williams, J. Appl. Phys. 110, 053110 (2011).

[20] J. D. Mar, J. J. Baumberg, X. L. Xu, A. C. Irvine, C. R. Stanley, and D. A. Williams, Phys. Rev. B 87, 155315 (2013).

[21] P. W. Fry, I. E. Itskevich, D. J. Mowbray, M. S. Skolnick, J. J. Finley, J. A. Barker, E. P. O’Reilly, L. R. Wilson, I. A. Larkin, P. A. Maksym, M. Hopkinson, M. Al-Khafaji, J. P. R. David, A. G. Cullis, G. Hill, and J. C. Clark, Phys. Rev. Lett. 84, 733 (2000).

[22] J. J. Finley, M. Sabathil, P. Vogl, G. Abstreiter, R. Oulton, A. I. Tartakovskii, D. J. Mowbray, M. S. Skolnick, S. L. Liew, A. G. Cullis, and M. Hopkinson, Phys. Rev. B 70, 201308(R) (2004).

[23] M. Grundmann, O. Stier, and D. Bimberg, Phys. Rev. B 52 , 11969 (1995).

[24] M. A. Cusack, P. R. Briddon, and M. Jaros, Phys. Rev. B 54, R2300 (1996).

[25] C. Pryor, Phys. Rev. B 57, 7190 (1998).

[26] J. Kim, L.-W. Wang, and A. Zunger, Phys. Rev. B 57, R9408 (1998).

[27] D. V. Bulaev and D. Loss, Phys. Rev. Lett. 95, 076805 (2005). 